\title{
RECONSTRUCTION OF CLUSTER MASS DISTRIBUTIONS: APPLICATION AND RESULTS FOR CL0939+4713
}

\author{
CAROLIN SEITZ \\ Max-Planck-Institut für Astrophysik \\ Postfach 1523, D-85740 Garching, Germany
}

\begin{abstract}
We show the reconstructed mass distribution of the cluster CL0939+4713 $\left(z_{d}=0.4\right)$ using data obtained with the WFPC2 instrument on HST. We find a remarkable correlation with the light distribution of the bright galaxies, which are probably cluster member galaxies. A bootstrapping analysis confirms this correlation, whereas an anti-correlation between the mass distribution and the faint galaxies - used for the mass reconstruction - is found. We give lower bounds on the mass inside the data field, and confirm that this cluster is indeed quite massive.
\end{abstract}

Because of the limited space, we do not describe the data analysis and the method of reconstruction, but refer the reader to the paper by Seitz et al. (1995, hereafter Paper I). We point out that due to the high redshift of the cluster $\left(z_{d}=0.4\right)$ the redshift distribution of the faint galaxies has to be accounted for in the reconstruction. In Figure 1 we show the reconstructed dimensionless mass density $\kappa$ for a source at 'infinity', assuming a mean redshift of $\langle z\rangle=0.8$ for the faint galaxies and using the invariance transformation derived in Paper I such that the minimum of the resulting $\kappa$-map is roughly zero. Comparing the mass distribution with the light distribution of the bright galaxies shown in Figure 2, we find that the position of the maximum mass density corresponds well with the position of the cluster center suggested by Dressler \& Gunn (1992). The secondary maximum corresponds to a group of bright cluster galaxies, and the minimum to a region where very few cluster galaxies are observed.

We performed a error bootstrap analysis, i.e., we generate a number of synthetic data sets by drawing $N$ galaxies at a time with replacement from the original data set consisting of $N=230$ galaxies with $m \in(24,25.5)$. For each of the synthetic data sets we obtain the mass-reconstruction and 


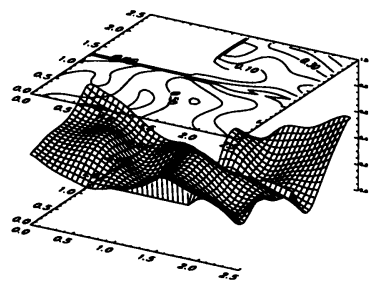

Figure 1. The reconstructed surface mass density of the cluster Cl0939. For the reconstruction we use about 230 galaxy images with $m \in(24,25.5)$ and assume that their mean redshift is $\langle z\rangle=0.8$. The field of view is $2^{\prime} .5$.

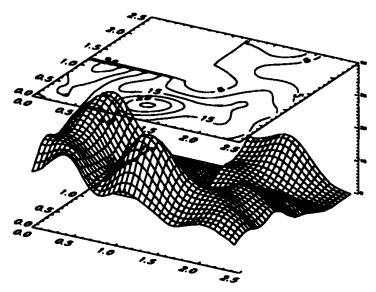
Figure 2. The gaus-
sian-smoothed light distribution of all bright galaxies with $m \in(18,22)$. We use a smoothing length of $s=0.3$. Dressler \& Gunn (1992) propose the cluster center to be in the upper left corner of the lower left CCD

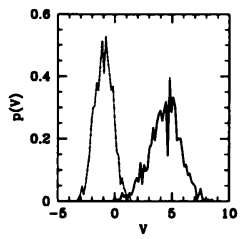

Figure 3. The distribution $p(V)$ calculated from reconstructed mass distributions for 900 bootstrapping data sets (see text). The solid line shows $p(V)$ for the bright galaxies $(m \in(18,22))$, the dotted one for the faint galaxies $(m \in(24,25.5))$

calculate the number $V:=\sum_{\text {galaxies }} \kappa_{\infty}\left(\vec{x}_{\text {galaxy }}\right)-\left\langle\kappa_{\infty}\right\rangle$, both for the faint galaxies used for the reconstruction and for the bright galaxies, which are mostly cluster member galaxies. From 900 simulations we find the distributions $p(V)$ shown in Figure 3. Clearly, a correlation between the bright galaxies and the reconstructed mass distribution shows up (solid line), whereas there is an anti-correlation for the faint galaxies (dotted line). This anti-correlation is most likely an effect of the magnification (anti)bias, and is expected from the slope of the observed number counts (see Paper I). Furthermore, we find that the main features of the reconstructed mass distribution shown in Figure 1, (i.e., the maximum of the mass distribution, the decrease to the right and the prominent minimum), are stable.

We confirm that this rich cluster is also quite massive; as a lower bound on the total mass inside the data field (side length about $1 h_{50}^{-1} \mathrm{Mpc}$ we find for a mean redshift $\langle z\rangle=0.6(0.8,1.0,1.5)$ about $M \geq 5.3 \times 10^{14} h_{50}^{-1} M_{\odot}$ $(3.6,3.0,2.3)$. These limits depend only slightly on the actual form of the assumed redshift distribution as shown in Paper I.

Acknowledgements: This work was supported by the "Sonderforschungsbereich 375-95 für Astro-Teilchenphysik" der Deutschen Forschungsgemeinschaft.

\section{References}

Dressler, A. \& Gunn, J.E., 1992, ApJS, 78, 1

Seitz, C., Kneib, J.P., Schneider, P. \& Seitz, S., 1995, in preparation (Paper I) 